\title{
EXISTENCE AND BOUNDEDNESS OF SOLUTIONS FOR SYSTEMS OF DIFFERENCE EQUATIONS WITH INFINITE DELAY
}

\author{
Juan J. Nieto, Abdelghani Ouahab and Mohammed A. Slimani \\ University of Santiago de Compostela, Spain and University of Sidi \\ Bel-Abbès, Algeria
}

\begin{abstract}
In this work we first establish some existence results followed by boundedness behavior and asymptotic behavior of solutions for systems of difference equations with infinite delay. Our approach is based on a Perov fixed point theorem in vector metric space. We apply our results to a system of Volterra difference equations.
\end{abstract}

\section{INTRODUCTION}

In this work, we are concerned with the existence and uniqueness of bounded solutions in some state space of sequences for a system of semilinear functional difference equations with infinite delay. Several aspects of the theory of functional difference equations can be understood as a proper generalization of the theory of ordinary difference equations. However, the fact that the state space for functional difference equations is infinite dimensional requires the development of methods and techniques coming from functional analysis (e.g., theory of semigroups of operators on Banach spaces, spectral theory, fixed point theory, etc.). Some important contributions to the study of the mathematical aspects of such equations have been undertaken in $[1,5,12]$ and the references therein.

2010 Mathematics Subject Classification. 34K45, 34A60.

Key words and phrases. Difference equations, fixed point, infinite delay, boundedness, asymptotic behavior, matrix convergent to zero. 
Abstract retarded functional difference equations in phase space have great importance in applications. Consequently, the theory of difference equations with infinite delay has drawn the attention of several authors. Qualitative analysis, discrete maximal regularity, exponential dichotomy, and periodicity have received much attention; see $[2-4,7-9,11,14-18,20-22,25,29,28$, $30,31]$. For more information on functional difference equations, we suggest also $[10,13,19,24,26,33]$.

In this paper, we consider the following system of linear functional difference equations

$$
\left\{\begin{array}{l}
x(n+1)=A_{1}\left(n, x_{n}, y_{n}\right), n \geq 0 \\
y(n+1)=A_{2}\left(n, x_{n}, y_{n}\right), n \geq 0
\end{array}\right.
$$

and its perturbation

$$
\left\{\begin{aligned}
x(n+1) & =A_{1}\left(n, x_{n}, y_{n}\right)+f_{1}\left(n, x_{n}, y_{n}\right), n \geq 0, \\
y(n+1) & =A_{2}\left(n, x_{n}, y_{n}\right)+f_{2}\left(n, x_{n}, y_{n}\right), n \geq 0, \\
x(0) & =\varphi \in \mathcal{B}, \\
y(0) & =\psi \in \mathcal{B},
\end{aligned}\right.
$$

where $A_{1}, A_{2}: \mathbb{Z}^{+} \times \mathcal{B} \times \mathcal{B} \rightarrow \mathbb{C}^{r}$ are bounded linear maps with respect to the variables $x_{n}$ and $y_{n} ; f_{1}, f_{2}$ are $\mathbb{C}^{r}$-valued functions defined on the product space $\mathbb{Z}^{+} \times X \times X$ under suitable conditions; $\mathcal{B}$ denotes an abstract phase space that we will explain briefly below, $X$ is an appropriate Banach space. The notation, $x$., denotes the $\mathcal{B}$-valued function defined by $n \rightarrow x_{n}$, where $x_{n}$ is the history function, which is defined by $x_{n}(m)=x(n+m)$ for all $m \in \mathbb{Z}^{-}$.

Next, we are concerned with the following homogeneous retarded linear functional equations

$$
\left\{\begin{array}{l}
x(n+1)=L_{1}\left(x_{n}, y_{n}\right), n \geq 0 \\
y(n+1)=L_{2}\left(x_{n}, y_{n}\right), n \geq 0
\end{array}\right.
$$

and its perturbation, along with initial conditions, defined by the semilinear difference equation with infinite delay

$$
\left\{\begin{aligned}
x(n+1) & =L_{1}\left(x_{n}, y_{n}\right)+g_{1}\left(n, x_{n}, y_{n}\right), n \geq 0, \\
y(n+1) & =L_{2}\left(x_{n}, y_{n}\right)+g_{2}\left(n, x_{n}, y_{n}\right), n \geq 0, \\
x(0) & =\varphi \in \mathcal{B}, \\
y(0) & =\psi \in \mathcal{B},
\end{aligned}\right.
$$

where $L_{1}, L_{2}: \mathcal{B} \times \mathcal{B} \rightarrow \mathbb{C}^{r}$ are bounded operators and $g_{1}, g_{2}: \mathbb{Z}^{+} \times \mathcal{B} \times \mathcal{B} \rightarrow \mathbb{C}^{r}$ are given functions.

This paper is organized as follows. In Sections 2 and 3 we will recall briefly some basic definitions and preliminary facts which will be used throughout the following sections. In Section 4 we give one of our main existence results for solutions of (1.4) and their boundedness, with the proof based on Perov's fixed point theorem. In Section 5, we examine the weighted boundedness and asymptotic behavior for solutions of (1.4). In Section 6, we present 
the $S$-asymptotic and $\omega$-periodic behavior of solutions of (1.4). Finally, in Section 7 an example is given to demonstrate the applicability of our results.

\section{Preliminaries}

Here we present notations and provide some auxiliary results that we will need in subsequent sections. The phase space $\mathcal{B}=\mathcal{B}\left(\mathbb{Z}^{-}, \mathbb{C}^{r}\right)$ is a Banach space with a norm denoted by $\|.\|_{\mathcal{B}}$ which is a subfamily of functions from $\mathbb{Z}^{-}$ into $\mathbb{C}^{r}$ and it is assumed to satisfy the following axioms.

Axiom (A): There are a positive constant $J$ and nonnegative functions $N($.) and $M($.$) on \mathbb{Z}^{+}$with the property that if $x: \mathbb{Z}^{+} \rightarrow \mathbb{C}^{r}$ is a function such that if $x_{0} \in \mathcal{B}$, then for all $n \in \mathbb{Z}^{+}$

(i) $x_{n} \in \mathcal{B}$;

(ii) $J\left|x_{n}\right| \leq\left\|x_{n}\right\|_{\mathcal{B}} \leq N(n) \sup _{0 \leq s \leq n}|x(s)|+M(n)\left\|x_{0}\right\|_{\mathcal{B}}$.

Denote by $B\left(\mathbb{Z}^{-}, \mathbb{C}^{r}\right)$ the set of bounded functions from $\mathbb{Z}^{-}$to $\mathbb{C}^{r}$.

Axiom (B): The inclusion map $i:\left(B\left(Z^{-}, \mathbb{C}^{r}\right),\|\cdot\|_{\infty}\right) \rightarrow\left(\mathcal{B},\|\cdot\|_{\mathcal{B}}\right)$ is continuous, i.e., there is a constant $d>0$ such that $\|\varphi\|_{\mathcal{B}} \leq d\|\varphi\|_{\infty}$ for all $\varphi \in \mathcal{B}\left(\mathbb{Z}^{-}, \mathbb{C}^{r}\right)$.

Hereafter, $\mathcal{B}$ will denote a phase space satisfying Axioms $(A)$ and $(B)$.

For any $n \geq \tau$ we define the bounded linear operator $U(n, \tau): \mathcal{B} \rightarrow \mathcal{B}$ by $U(n, \tau) \varphi=x_{n}(\tau, \varphi, 0)$ for $\varphi \in \mathcal{B}$, where $x(\cdot, \tau, \varphi, 0)$ denotes the solution of the homogeneous linear system (1.1). The operator $U(n, \tau)$ is called the solution operator of the homogeneous linear system (1.1).

Definition 2.1 ([11]). We say that Equation (1.1) (or its solution operator $U(n, \tau), n, \tau \in \mathbb{Z}^{+}$) has an exponential dichotomy on $\mathcal{B}$ with data $(\alpha, K, P(\cdot))$, if the solution operator $U(n, \tau)$ satisfies the following property: there are positive constants $\alpha, K$, and a projection operator $P(n), n \in \mathbb{Z}^{+}$, in $\mathcal{B}$, such that if $Q(n)=I-P(n)$, where $I$ is the identity operator, then:

(i) $U(n, \tau) P(\tau)=P(n) U(n, \tau), n \geq \tau$,

(ii) the restriction $U(n, \tau) \mid$ Range $(Q(\tau)), n \geq \tau$, is an isomorphism from Range $(Q(\tau))$ onto Range $(Q(n))$, and then we define $(U(\tau, n))$ as its inverse mapping,

(iii) $\|U(n, \tau) \varphi\|_{\mathcal{B}} \leq K e^{-\alpha(n-\tau)}\|\varphi\|_{\mathcal{B}}, n \geq \tau, \varphi \in P(\tau) \mathcal{B}$,

(iv) $\|U(n, \tau) \varphi\|_{\mathcal{B}} \leq K e^{\alpha(n-\tau)}\|\varphi\|_{\mathcal{B}}, \tau>n, \varphi \in Q(\tau) \mathcal{B}$.

We denote by $\Gamma(n, s)$ the Green function associated with (1.1), that is,

$$
\Gamma(t, s)= \begin{cases}U(n, s+1) P(s+1) & n-1 \geq s \\ -U(n, s+1) Q(s+1) & s>n-1\end{cases}
$$

Denote by $X$ the Banach space of all bounded functions $\eta: \mathbb{Z}^{+} \rightarrow \mathcal{B}$ endowed with the norm

$$
\|\eta\|=\sup _{n \geq 0}\|\eta\|_{\mathcal{B}} .
$$


Throughout this paper, for any number $1 \leq p<\infty$, we consider the following spaces:

$$
\begin{aligned}
l^{p}\left(\mathbb{Z}^{+}, \mathcal{B}\right) & =\left\{\xi: \mathbb{Z}^{+} \rightarrow \mathcal{B} \mid\|\xi(n)\|_{p}^{p}=\sum_{n=0}^{\infty}\|\xi(n)\|_{\mathcal{B}}^{p}<\infty\right\}, \\
l^{\infty}\left(\mathbb{Z}^{+}, \mathcal{B}\right) & =\left\{\xi: \mathbb{Z}^{+} \rightarrow \mathcal{B} \mid\|\xi\|_{\infty}=\sup _{n \in \mathbb{Z}^{+}}\|\xi(n)\|_{\mathcal{B}}<\infty\right\}, \\
l_{\beta}^{\infty}\left(\mathbb{Z}^{+}, \mathcal{B}\right) & =\left\{\xi: \mathbb{Z}^{+} \rightarrow \mathcal{B} \mid\|\xi\|_{\beta}=\sup _{n \in Z^{+}}\|\xi(n)\|_{\mathcal{B}} e^{-\beta n}<\infty\right\}, \\
l_{\beta}^{1}\left(\mathbb{Z}^{+}, \mathbb{C}^{r}\right) & =\left\{\varphi: \mathbb{Z}^{-} \rightarrow \mathbb{C}^{r}\left|\|\varphi\|_{1, \beta}=\sum_{n=0}^{\infty}\right| \varphi(n) \mid e^{-\beta n}<\infty\right\}, \\
l^{p}\left(\mathbb{Z}^{+}, \mathbb{C}^{r}\right) & =\left\{\varphi: \mathbb{Z}^{+} \rightarrow \mathbb{C}^{r} /\|\varphi\|_{p}^{p}=\sum_{n=0}^{\infty}|\varphi(n)|^{p}<\infty\right\} .
\end{aligned}
$$

Lemma 2.2. Let $\tau \in \mathbb{Z}^{+}$. Assume that the function $z:[\tau, \infty) \rightarrow \mathcal{B}$ satisfies the relation

$$
z(n)=U(n, \tau) z(\tau)+\sum_{s=\tau}^{n-1} U(n, s+1) E^{0}(P(s)), \quad n \geq \tau
$$

where

and define a function $y: \mathbb{Z} \rightarrow \mathbb{C}^{r}$ by

$$
E^{0}(t)=\left\{\begin{array}{l}
I d, \quad \text { the unit matrix }, t=0, \\
0, \quad \text { the zero matrix }, t<0
\end{array}\right.
$$

$$
y(n)= \begin{cases}(z(n))(0), & n \geq \tau, \\ (z(\tau))(n-\tau), & n<\tau .\end{cases}
$$

Then $y(n)$ satisfies the equation

$$
y(n+1)=L\left(n, y_{n}\right)+P(n), n \geq \tau,
$$

together with relation $y_{n}=z(n), \quad n \geq \tau$.

DEFinition 2.3 ([17]). We say that system (1.1) has discrete maximal regularity if, for each $h_{1}, h_{2} \in l^{p}\left(\mathbb{Z}^{+}, \mathbb{C}^{r}\right)(1 \leq p<\infty)$ and each $\varphi, \psi \in P(0) \mathcal{B}$, the solution $z$ of the boundary value problem

$$
\left\{\begin{array}{l}
z(n+1)=A_{1}\left(n, z_{n}, \tilde{z}_{n}\right)+h_{1}(n), n \geq 0 \\
\tilde{z}(n+1)=A_{2}\left(n, z_{n}, \tilde{z}_{n}\right)+h_{2}(n), n \geq 0 \\
P(0) z(0)=\varphi \in \mathcal{B} \\
P(0) \tilde{z}(0)=\psi \in \mathcal{B}
\end{array}\right.
$$

satisfies z., $\tilde{z} . \in l^{p}\left(\mathbb{Z}^{+}, \mathcal{B}\right)$.

THEOREM 2.4 ([11]). Assume that system (1.1) has an exponential dichotomy on $\mathcal{B}$ with data $(\alpha, K, P(\cdot))$. Then system $(1.2)$ has discrete maximal regularity. 
Theorem 2.5. Assume that system (1.1) has an exponential dichotomy with data $(\alpha, K, P(\cdot))$. Then, for any $h_{1}, h_{2} \in l^{p}\left(\mathbb{Z}^{+}, \mathbb{C}^{r}\right)(1 \leq p<\infty)$ and each $\varphi, \psi \in P(0) \mathcal{B}$, the boundary value problem (2.6) has a unique solution $(z, \tilde{z})$ so that $z$. $\tilde{z} . \in l^{p}\left(\mathbb{Z}^{+}, \mathcal{B}\right)$.

Theorem 2.6 (Exponential boundedness of the solution operator, [11]). Assume that

$\left(H_{1}\right)\left\{A_{1}(n, \cdot, \cdot)\right\}$ and $\left\{A_{2}(n, \cdot, \cdot)\right\}$ are uniformly bounded sequences of bounded linear operators mapping $\mathcal{B} \times \mathcal{B}$ into $\mathbb{C}^{r}$. There are constants $M_{i}, N_{i}>1, i=1,2$ such that

$\left|A_{1}(n, \varphi, \psi)\right| \leq M_{1}\|\varphi\|_{\mathcal{B}}+N_{1}\|\psi\|_{\mathcal{B}}$, for all $n \in \mathbb{Z}^{+}$and $\varphi, \psi \in \mathcal{B}$,

and

$\left|A_{2}(n, \varphi, \psi)\right| \leq M_{2}\|\varphi\|_{\mathcal{B}}+N_{2}\|\psi\|_{\mathcal{B}}$, for all $n \in \mathbb{Z}^{+}$and $\varphi, \psi \in \mathcal{B}$.

$\left(H_{2}\right)$ The functions $N($.$) and M($.$) given in Axiom (A) are bounded.$

Then, there are positive constants $\lambda$ and $\delta$ such that

$$
\|U(n, m)\|_{\mathcal{B}} \leq \lambda e^{\delta(n-m)}, n \geq m \geq 0 .
$$

Proposition 2.7 ([11]). Under the conditions $\left(H_{1}\right)-\left(H_{2}\right)$, if system $(1.1)$ has an exponential dichotomy with data $(\alpha, K, P()$.$) , then the following haolds.$

(i) $\sup _{n \in \mathbb{Z}^{+}}\|P(n)\|_{\mathcal{B}}<\infty$.

(ii) Range $(P(n))=\left\{\varphi \in \mathcal{B}: e^{-\eta(n-m)} U(n, m) \varphi\right.$ is bounded for $\left.n \geq m\right\}$ for any $0<\eta<\alpha$.

(iii) Let $\widehat{P}(0)$ be a projection such that Range $(\widehat{P}(0))=\operatorname{Range}(P(0))$. Then (1.2) has an exponential dichotomy on $\mathbb{Z}^{+}$with data $(\alpha, \widehat{K}, \widehat{P}(\cdot))$, where

$$
\begin{aligned}
& \widehat{P}(n)=P(n)+U(n, 0) \widehat{P}(0) U(0, n) Q(n), \\
& \widehat{K}=\left(K+K^{2}\|\widehat{P}(0)\|_{\mathcal{B}}\right) \sup _{m \geq 0}\left(1+\|P(n)\|_{\mathcal{B}}\right) .
\end{aligned}
$$

In addition, we have

$$
\sup _{m \geq 0}\|\widehat{P}(m)\|_{\mathcal{B}} \leq\left(1+K^{2}\|\widehat{P}(0)-P(0)\|_{\mathcal{B}}\right) \sup _{m \geq 0}\left(1+\|P(m)\|_{\mathcal{B}}\right) .
$$

Also one has

$$
\widehat{P}(n)=P(n)+o(1) \text {, as } n \rightarrow \infty .
$$

Definition $2.8([23])$. A sequence $\xi \in l^{\infty}\left(\mathbb{Z}^{+}, \mathcal{B}\right)$ is called (discrete) $S$ asymptotically $\omega$-periodic if there is $\omega \in \mathbb{Z}^{+} \backslash\{0\}$ such that $\lim _{n \rightarrow \infty}(\xi(n+\omega)-$ $\xi(n))=0$. In this case we say that $\omega$ is an asymptotic period of $\xi$.

In this work the notation $S A P_{\omega}(\mathcal{B})$ stands for the subspace of $l^{\infty}\left(\mathbb{Z}^{+}, \mathcal{B}\right)$ consisting of all the (discrete) $S$-asymptotically $\omega$-periodic sequences. From [23], $S A P_{\omega}(\mathcal{B})$ is a Banach space. 
Definition 2.9 ([23]). A strongly continuous function $F: \mathbb{Z}^{+} \rightarrow \mathcal{L}(\mathcal{B})$ is said to be strongly $S$-asymptotically periodic if, for each $\varphi \in \mathcal{B}$, there is $\omega_{\varphi} \in \mathbb{Z}^{+} \backslash\{0\}$ such that $F(.) \varphi$ is $S$-asymptotically $\omega_{\varphi}$-periodic.

Definition 2.10 ([23]). A continuous function $g: \mathbb{Z}^{+} \times \mathcal{B} \rightarrow \mathbb{C}^{r}$ is said to be uniformly $S$-asymptotically $\omega$-periodic on bounded sets if, for every bounded subset $B$ of $\mathcal{B}$, the set $\left\{g(n, \varphi): n \in \mathbb{Z}^{+}, \varphi \in B\right\}$ is bounded and $\lim _{n \rightarrow \infty}(g(n, \varphi)-g(n+\omega, \varphi))=0$ uniformly on $\varphi \in B$.

Definition 2.11 ([23]). A function $g: \mathbb{Z}^{+} \times \mathcal{B} \rightarrow \mathbb{C}^{r}$ is called uniformly asymptotically continuous on bounded sets if, for every $\epsilon>0$ and every bounded subset $B$ of $\mathcal{B}$, there are $K_{\epsilon, B} \geq 0$ and $\delta_{\epsilon, B} \geq 0$ such that $|g(n, \varphi)-g(n, \psi)|<\epsilon$, for all $n \geq K_{\epsilon, B}$ and all $\varphi, \psi \in B$ with $\|\varphi-\psi\|<\delta_{\epsilon, B}$.

Lemma $2.12([23])$. Let $g: \mathbb{Z}^{+} \times \mathcal{B} \rightarrow \mathbb{C}^{r}$ be uniformly S-asymptotically $\omega$ periodic on bounded sets and asymptotically uniformly continuous on bounded sets. Let $\xi: \mathbb{Z}^{+} \rightarrow \mathcal{B}$ be a discrete $S$-asymptotically $\omega$-periodic function. Then the function $g(\cdot, \xi(\cdot))$ is discrete $S$-asymptotically $\omega$-periodic.

\section{Fixed POINT RESUlts}

In this short section, we introduce notations and definitions which are used throughout this paper.

Definition 3.1. Let $X$ be a nonempty set and consider the space $\mathbb{R}_{+}^{m}$ endowed with the usual component-wise partial order. The mapping $d: X \times X \rightarrow$ $\mathbb{R}_{+}^{m}$, which satisfies all the usual axioms of the metric, is called a generalized metric in Perov's sense and $(X, d)$ is called a vector metric space.

Let $(X, d)$ be a vector metric space in Perov's sense. For $r:=$ $\left(r_{1}, \ldots, r_{m}\right) \in \mathbb{R}_{+}^{m}$, we denote by

$$
B\left(x_{0}, r\right)=\left\{x \in X: d\left(x_{0}, x\right)<r\right\}
$$

the open ball centered in $x_{0}$ with radius $r$ and by

$$
\overline{B\left(x_{0}, r\right)}=\left\{x \in X: d\left(x_{0}, x\right) \leq r\right\}
$$

the closed ball centered at $x_{0}$ with radius $r$.

In the case of vector metric spaces in the sense of Perov, the notions of convergent sequence, Cauchy sequence, completeness, and open and closed subsets are similar to those for usual metric spaces.

If $v, r \in \mathbb{R}^{m}, v:=\left(v_{1}, \ldots, v_{m}\right)$ and $r:=\left(r_{1}, \ldots, r_{m}\right)$, then by $v \leq r$ we mean $v_{i} \leq r_{i}$ for each $i \in\{1, \ldots, m\}$ and by $v<r$ we mean $v_{i}<r_{i}$ for each $i \in\{1, \ldots, m\}$. Also $|v|:=\left(\left|v_{1}\right|, \ldots,\left|v_{m}\right|\right)$ and $\max (u, v):=$ $\left(\max \left(u_{1}, v_{1}\right), \ldots, \max \left(u_{m}, v_{m}\right)\right)$. If $c \in \mathbb{R}$, then $v \leq c$ means $v_{i} \leq c$ for each $i \in\{1, \ldots, m\}$. 
Throughout this paper we denote by $\mathcal{M}_{m, m}\left(\mathbb{R}_{+}\right)$the set of all $m \times m$ matrices with positive elements, by $\Theta$ the zero $m \times m$ matrix, and by $I d$ the identity $m \times m$ matrix.

Definition 3.2. A square matrix $M \in \mathcal{M}_{m, m}\left(\mathbb{R}_{+}\right)$is said to be convergent to zero if

$$
M^{k} \rightarrow \Theta \text { as } k \rightarrow \infty
$$

LEMMA 3.3 ([32]). Let $M$ be a square matrix of nonnegative numbers. Then the following assertions are equivalent:

1. $M$ is convergent to zero;

2. the matrix Id $-M$ is non-singular and

$$
(I d-M)^{-1}=I d+M+M^{2}+\cdots+M^{k}+\cdots ;
$$

3. $\|\lambda\|<1$ for every $\lambda \in \mathbb{C}$ with $\operatorname{det}(M-\lambda I d)=0$;

4. $(I d-M)$ is non-singular and $(I d-M)^{-1}$ has nonnegative elements.

Definition 3.4. We say that a non-singular matrix $A=\left(a_{i j}\right)_{1 \leq i, j \leq m} \in$ $\mathcal{M}_{m \times m}(\mathbb{R})$ has the absolute value property if

$$
A^{-1}|A| \leq I d
$$

where

$$
|A|=\left(\left|a_{i j}\right|\right)_{1 \leq i, j \leq m} \in \mathcal{M}_{m \times m}\left(\mathbb{R}_{+}\right) .
$$

Some examples of matrices convergent to zero are the following:

1. $A=\left(\begin{array}{cc}a & 0 \\ 0 & b\end{array}\right)$, where $a, b \in \mathbb{R}_{+}$and $\max (a, b)<1$.

2. $A=\left(\begin{array}{cc}a & -c \\ 0 & b\end{array}\right)$, where $a, b, c \in \mathbb{R}_{+}$and $a+b<1, c<1$.

3. $A=\left(\begin{array}{cc}a & -a \\ b & -b\end{array}\right)$, where $a, b \in \mathbb{R}_{+}$and $|a-b|<1, a>1, b>0$.

TheOREM $3.5([27])$. Let $(X, d)$ be a complete vector metric space with $d: X \times X \rightarrow \mathbb{R}^{m}$ and let $N: X \rightarrow X$ be such that

$$
d(N(x), N(y)) \leq M d(x, y),
$$

for all $x, y \in X$ and some square matrix $M$ of nonnegative numbers. If the matrix $M$ is convergent to zero, that is, $M^{k} \rightarrow 0$ as $k \rightarrow \infty$, then $N$ has a unique fixed point $x_{*} \in X$,

$$
d\left(N^{k}\left(x_{0}\right), x_{*}\right) \leq M^{k}(I d-M)^{-1} d\left(N\left(x_{0}\right), x_{0}\right)
$$

for every $x_{0} \in X$ and $k \geq 1$. 


\section{Boundedness of Solutions}

In this section, we are concerned with the study of the existence of bounded solutions for the semilinear difference equation with infinite delay via discrete maximal regularity.

Theorem 4.1. Assume that system (1.1) has exponential dichotomy on $\mathcal{B}$, and in addition to conditions $\left(H_{1}\right)$ and $\left(H_{2}\right)$, suppose that the following conditions hold:

$\left(H_{3}\right)$ the functions $f_{i}(n, \cdot, \cdot): l^{p}\left(\mathbb{Z}^{+}, \mathcal{B}\right) \times l^{p}\left(\mathbb{Z}^{+}, \mathcal{B}\right) \rightarrow \mathbb{C}^{r}, i=1,2$ satisfy, for all $x, y, \bar{x}, \bar{y} \in l^{p}\left(\mathbb{Z}^{+}, \mathcal{B}\right)$ and $n \in \mathbb{Z}^{+}$,

$$
\left|f_{1}(n, x, y)-f_{1}(n, \bar{x}, \bar{y})\right| \leq a_{1}(n)\|x-\bar{x}\|_{p}+b_{1}(n)\|y-\bar{y}\|_{p}
$$

and

$$
\left|f_{2}(n, x, y)-f_{2}(n, \bar{x}, \bar{y})\right| \leq a_{2}(n)\|x-\bar{x}\|_{p}+b_{2}(n)\|y-\bar{y}\|_{p}
$$

where $a_{i}, b_{i} \in l^{p}\left(\mathbb{Z}^{+}\right), i=1,2$,

$\left(H_{4}\right) f_{1}(\cdot, 0,0), f_{2}(\cdot, 0,0) \in l^{p}\left(\mathbb{Z}^{+}, \mathbb{C}^{r}\right)$,

$\left(H_{5}\right)$ the matrix $M \in \mathcal{M}_{2 \times 2}\left(\mathbb{R}_{+}\right)$such that

$$
M=2 d K\left(1-e^{-\alpha}\right)^{-1} \sup _{n \in \mathbb{Z}^{+}}\left(1+\|P(n)\|_{\mathcal{B}}\right)\left(\begin{array}{cc}
\left\|a_{1}\right\|_{p} & \left\|b_{1}\right\|_{p} \\
\left\|a_{2}\right\|_{p} & \left\|b_{2}\right\|_{p}
\end{array}\right)
$$

converges to zero.

Then, for each $\varphi, \psi \in P(0) \mathcal{B}$ there is a unique bounded solution $(x, y)$ of system (1.2) with $P(0) x_{0}=\varphi, P(0) y_{0}=\psi$, such that $(x, y.) \in l^{p}\left(\mathbb{Z}^{+}, \mathcal{B}\right) \times$ $l^{p}\left(\mathbb{Z}^{+}, \mathcal{B}\right)$.

Proof. Let $\xi, \eta$ be sequences in $l^{p}\left(\mathbb{Z}^{+}, \mathcal{B}\right)$. Using conditions $\left(H_{3}\right)$ and $\left(H_{4}\right)$ we obtain that the function $F(\cdot)=f_{1}(\cdot, \xi, \eta)$ is in $l^{p}\left(\mathbb{Z}^{+}, \mathbb{C}^{r}\right)$, and we have

$$
\begin{aligned}
\|F\|_{p}^{p} & =\sum_{n=0}^{\infty}\left|f_{1}(n, \xi, \eta)\right|^{p} \\
& \leq \sum_{n=0}^{\infty}\left(\left|f_{1}(n, \xi, \eta)-f_{1}(n, 0,0)\right|+\left|f_{1}(n, 0,0)\right|\right)^{p} \\
& \leq 2^{p} \sum_{n=0}^{\infty}\left|f_{1}(n, \xi, \eta)-f_{1}(n, 0,0)\right|^{p}+2^{p} \sum_{n=0}^{\infty}\left|f_{1}(n, 0,0)\right|^{p} \\
& \leq 2^{p} \sum_{n=0}^{\infty}\left(a_{1}^{p}(n)\|\xi\|_{\mathcal{B}}^{p}+b_{1}^{p}(n)\|\eta\|_{\mathcal{B}}^{p}\right)+2^{p}\left\|f_{1}(n, 0,0)\right\|_{p}^{p} .
\end{aligned}
$$

Hence

$$
\|F\|_{p} \leq 2\left(\left\|a_{1}\right\|_{p}\|\xi\|_{p}+\left\|b_{1}\right\|_{p}\|\eta\|_{p}+\left\|f_{1}(\cdot, 0,0)\right\|_{p}\right) .
$$


Similarly we obtain that the function $G(\cdot)=f_{2}(\cdot, \xi, \eta)$ is in $l^{p}\left(\mathbb{Z}^{+}, \mathbb{C}^{r}\right)$, and

$$
\begin{aligned}
\|G\|_{p}^{p} & =\sum_{n=0}^{\infty}\left|f_{2}(n, \xi, \eta)\right|^{p} \\
& \leq \sum_{n=0}^{\infty}\left(\left|f_{2}(n, \xi, \eta)-f_{2}(n, 0,0)\right|+\left|f_{2}(n, 0,0)\right|\right)^{p} \\
& \leq 2^{p} \sum_{n=0}^{\infty}\left|f_{2}(n, \xi, \eta)-f_{2}(n, 0,0)\right|^{p}+2^{p} \sum_{n=0}^{\infty}\left|f_{2}(n, 0,0)\right|^{p} \\
& \leq 2^{p} \sum_{n=0}^{\infty}\left(a_{2}^{p}(n)\|\xi\|_{\mathcal{B}}^{p}+b_{2}^{p}(n)\|\eta\|_{\mathcal{B}}^{p}\right)+2^{p}\left\|f_{2}(n, 0,0)\right\|_{p}^{p} .
\end{aligned}
$$

Hence

$$
\|G\|_{p} \leq 2\left(\left\|a_{2}\right\|_{p}\|\xi\|_{p}+\left\|b_{2}\right\|_{p}\|\eta\|_{p}+\left\|f_{2}(\cdot, 0,0)\right\|_{p}\right)
$$

If $\varphi, \psi \in P(0) \mathcal{B}$, then by Theorem 2.4, system (1.2) has discrete maximal regularity, and so the Cauchy system

$$
\left\{\begin{aligned}
z(n+1) & =A_{1}\left(n, z_{n}, \widetilde{z}_{n}\right)+F(n), n \in \mathbb{Z}^{+} \\
\widetilde{z}(n+1) & =A_{2}\left(n, z_{n}, \widetilde{z}_{n}\right)+G(n), n \in \mathbb{Z}^{+} \\
P(0) z_{0} & =\varphi \\
P(0) \widetilde{z}_{0} & =\psi
\end{aligned}\right.
$$

has a unique solution $(z, \widetilde{z}) \in l^{p}\left(\mathbb{Z}^{+}, \mathcal{B}\right) \times l^{p}\left(\mathbb{Z}^{+}, \mathcal{B}\right)$, which is given by

$$
z_{n}=H_{1}(\xi(n), \eta(n))=U(n, 0) P(0) \varphi+\sum_{s=0}^{\infty} \Gamma(n, s) f_{1}(s, \xi, \eta)
$$

and

$$
\widetilde{z}_{n}=H_{2}(\xi(n), \eta(n))=U(n, 0) P(0) \psi+\sum_{s=0}^{\infty} \Gamma(n, s) f_{2}(s, \xi, \eta)
$$

We now show that the operator $H: l^{p}\left(\mathbb{Z}^{+}, \mathcal{B}\right) \times l^{p}\left(\mathbb{Z}^{+}, \mathcal{B}\right) \rightarrow l^{p}\left(\mathbb{Z}^{+}, \mathcal{B}\right) \times$ $l^{p}\left(\mathbb{Z}^{+}, \mathcal{B}\right)$ has a unique fixed point, defined as follows:

$H(\xi(n), \eta(n))=\left(H_{1}(\xi(n), \eta(n)), H_{2}(\xi(n), \eta(n))\right), \quad(\xi, \eta) \in l^{p}\left(\mathbb{Z}^{+}, \mathcal{B}\right) \times l^{p}\left(\mathbb{Z}^{+}, \mathcal{B}\right)$ 
Let $\xi_{1}, \eta_{1}, \xi_{2}, \eta_{2} \in l^{p}\left(\mathbb{Z}^{+}, \mathcal{B}\right)$. We have that

$$
\begin{aligned}
&\left\|H_{1}\left(\xi_{1}, \eta_{1}\right)-H_{1}\left(\xi_{2}, \eta_{2}\right)\right\|_{p} \\
&=\left[\sum_{n=0}^{\infty}\left\|\sum_{s=0}^{\infty} \Gamma(n, s)\left[f_{1}\left(s, \xi_{1}, \eta_{1}\right)-f_{1}\left(s, \xi_{2}, \eta_{2}\right)\right]\right\|_{\mathcal{B}}^{p}\right]^{\frac{1}{p}} \\
& \leq d K \sup _{n \in \mathbb{Z}^{+}}\left(1+\|P(n)\|_{\mathcal{B}}\right) \\
& \times\left[\sum_{n=0}^{\infty}\left(\sum_{s=0}^{\infty} e^{-\alpha|n-(s+1)|}\left[a_{1}(s)\left\|\xi_{1}-\xi_{2}\right\|_{\mathcal{B}}+b_{1}(s)\left\|\eta_{1}-\eta_{2}\right\|_{\mathcal{B}}\right]\right)^{p}\right]^{\frac{1}{p}} \\
& \leq 2 d K \sup _{n \in \mathbb{Z}^{+}}\left(1+\|P(n)\|_{\mathcal{B}}\right) \\
& \times \sum_{n=0}^{\infty} e^{-\alpha n} \sum_{s=0}^{\infty} e^{\alpha(s+1)}\left(\left\|a_{1}\right\|\left\|_{p}\right\| \xi_{1}-\xi_{2}\left\|_{p}+\right\| b_{1}\left\|_{p}\right\| \eta_{1}-\eta_{2} \|_{p}\right) \\
& \leq 2 d K\left(1-e^{-\alpha}\right)^{-1} \sup _{n \in Z^{+}}\left(1+\|P(n)\|_{\mathcal{B}}\right) \\
& \times\left(\left\|a_{1}\right\|_{p}\left\|\xi_{1}-\xi_{2}\right\|_{p}+\left\|b_{1}\right\|_{p}\left\|\eta_{1}-\eta_{2}\right\|_{p}\right) .
\end{aligned}
$$

Hence

$$
\begin{aligned}
\left\|H_{1}\left(\xi_{1}, \eta_{1}\right)-H_{1}\left(\xi_{2}, \eta_{2}\right)\right\|_{p} \leq & 2 d K\left(1-e^{-\alpha}\right)^{-1} \sup _{n \in \mathbb{Z}^{+}}\left(1+\|P(n)\|_{\mathcal{B}}\right) \\
& \times\left(\left\|a_{1}\right\|_{p}\left\|\xi_{1}-\xi_{2}\right\|_{p}+\left\|b_{1}\right\|_{p}\left\|\eta_{1}-\eta_{2}\right\|_{p}\right) .
\end{aligned}
$$

Similarly

$$
\begin{aligned}
\left\|H_{2}\left(\xi_{1}, \eta_{1}\right)-H_{2}\left(\xi_{2}, \eta_{2}\right)\right\|_{p} \leq & 2 d K\left(1-e^{-\alpha}\right)^{-1} \sup _{n \in \mathbb{Z}^{+}}\left(1+\|P(n)\|_{\mathcal{B}}\right) \\
& \times\left(\left\|a_{2}\right\|_{p}\left\|\xi_{1}-\xi_{2}\right\|_{p}+\left\|b_{2}\right\|\left\|_{p}\right\| \eta_{1}-\eta_{2} \|_{p}\right) .
\end{aligned}
$$

Then

$$
\begin{aligned}
\left\|H\left(\xi_{1}, \eta_{1}\right)-H\left(\xi_{2}, \eta_{2}\right)\right\|_{p} \leq & 2 d K\left(1-e^{-\alpha}\right)^{-1} \sup _{n \in \mathbb{Z}^{+}}\left(1+\|P(n)\|_{\mathcal{B}}\right) \\
& \times\left(\begin{array}{cc}
\left\|a_{1}\right\|_{p} & \left\|b_{1}\right\|_{p} \\
\left\|a_{2}\right\|_{p} & \left\|b_{2}\right\|_{p}
\end{array}\right)\left(\begin{array}{c}
\left\|\xi_{1}-\xi_{2}\right\|_{p} \\
\left\|\eta_{1}-\eta_{2}\right\|_{p}
\end{array}\right) .
\end{aligned}
$$

By $\left(H_{5}\right)$ and Theorem 3.5, it follows that $H$ has a unique fixed point $(\xi, \eta) \in$ $l^{p}\left(\mathbb{Z}^{+}, \mathcal{B}\right) \times l^{p}\left(\mathbb{Z}^{+}, \mathcal{B}\right)$. 
Let $(\xi, \eta)$ be the unique fixed point of $H$. Then we have

$$
\begin{aligned}
\|\xi\|_{p}= & {\left[\sum_{n=0}^{\infty}\left\|U(n, 0) P(0) \varphi+\sum_{s=0}^{\infty} U(n, s+1) f_{1}(s, \xi, \eta)\right\|_{\mathcal{B}}^{p}\right]^{\frac{1}{p}} } \\
\leq & {\left[\sum_{n=0}^{\infty}\|U(n, 0) P(0) \varphi\|_{\mathcal{B}}^{p}\right]^{\frac{1}{p}}+\left[\sum_{n=0}^{\infty}\left\|\sum_{s=0}^{\infty} U(n, s+1) f_{1}(s, \xi, \eta)\right\|_{\mathcal{B}}\right]^{\frac{1}{p}} } \\
\leq & K\left[\sum_{j=0}^{\infty} e^{-\alpha p j}\right]^{1 / p}\|\varphi\|_{\mathcal{B}} \\
& +2 d K \sup _{m \geq 0}\left(1+\|P(m)\|_{\mathcal{B}}\right)\left(1-e^{-\alpha}\right)^{-1}\left[\sum_{s=0}^{\infty}\left|f_{1}(s, \xi, \eta)\right|^{p}\right]^{1 / p} \\
\leq & K\left(1-e^{-\alpha}\right)^{-1}\|\varphi\|_{\mathcal{B}} \\
& +2 d K \sup _{m \geq 0}\left(1+\|P(m)\|_{\mathcal{B}}\right)\left(1-e^{-\alpha}\right)^{-1} \\
& \times\left(\left\|a_{1}\right\|_{p}\|\xi\|_{p}+\left\|b_{1}\right\|_{p}\|\eta\|_{p}+\left\|f_{1}(., 0)\right\|_{p}\right)
\end{aligned}
$$

and

$$
\begin{aligned}
\|\eta\|_{p} \leq & \left(1-e^{-\alpha}\right)^{-1}\|\psi\|_{\mathcal{B}} \\
+ & 2 d K \sup _{m \geq 0}\left(1+\|P(m)\|_{\mathcal{B}}\right)\left(1-e^{-\alpha}\right)^{-1} \\
& \quad \times\left(\left\|a_{2}\right\|_{p}\|\xi\|_{p}+\left\|b_{2}\right\|_{p}\|\eta\|_{p}+\left\|f_{2}(., 0)\right\|_{p}\right),
\end{aligned}
$$

whence

$$
\begin{aligned}
\left(\begin{array}{c}
\|\xi\|_{p} \\
\|\eta\|_{p}
\end{array}\right) \leq & 2 K d \sup _{m \geq 0}\left(1+\|P(m)\|_{\mathcal{B}}\left(1-e^{-\alpha}\right)^{-1}\left(\begin{array}{cc}
\left\|a_{1}\right\|_{p} & \left\|b_{1}\right\|_{p} \\
\left\|a_{2}\right\|_{p} & \left\|b_{2}\right\|_{p}
\end{array}\right)\left(\begin{array}{c}
\|\xi\|_{p} \\
\|\eta\|_{p}
\end{array}\right)\right. \\
+ & K\left(1-e^{-\alpha}\right)^{-1}\left(\begin{array}{c}
\|\varphi\|_{p} \\
\|\psi\|_{p}
\end{array}\right)+2 d K \sup _{m \geq 0}\left(1+\|P(m)\|_{\mathcal{B}}\right) \\
& \times\left(1-e^{-\alpha}\right)^{-1}\left(\begin{array}{c}
\left\|f_{1}(\cdot, 0)\right\|_{p} \\
\left\|f_{2}(\cdot, 0)\right\|_{p}
\end{array}\right) .
\end{aligned}
$$

Then

$$
\begin{aligned}
&\left(\begin{array}{c}
\|\xi\|_{p} \\
\|\eta\|_{p}
\end{array}\right) \leq(I d-M)^{-1}\left[K\left(1-e^{-\alpha}\right)^{-1}\left(\begin{array}{c}
\|\varphi\|_{p} \\
\|\psi\|_{p}
\end{array}\right)\right. \\
&\left.+2 d K \sup _{m \geq 0}\left(1+\|P(m)\|_{\mathcal{B}}\right)\left(1-e^{-\alpha}\right)^{-1}\left(\begin{array}{c}
\left\|f_{1}(\cdot, 0)\right\|_{p} \\
\left\|f_{2}(\cdot, 0)\right\|_{p}
\end{array}\right)\right] .
\end{aligned}
$$




\section{Weighted BOUNDEDNESS AND ASYMPtotiC BEHAVIOR}

We have the following result about weighted bounded solutions.

Theorem 5.1. Assume that conditions $\left(H_{1}\right)-\left(H_{2}\right)$ hold. Let $\lambda$ and $\delta$ be the constants of Theorem 2.6. In addition, suppose that the following conditions hold:

$\left(C_{1}\right)$ the functions $f_{i}(n, \cdot, \cdot): \mathcal{B} \times \mathcal{B} \rightarrow \mathbb{C}^{r}, i=1,2$ satisfy, for all $x, y, \bar{x}, \bar{y} \in$ $\mathcal{B}$ and $n \in \mathbb{Z}^{+}$,

$$
\left|f_{1}(n, x, y)-f_{1}(n, \bar{x}, \bar{y})\right| \leq a_{1}(n)\|x-\bar{x}\|_{\mathcal{B}}+b_{1}(n)\|y-\bar{y}\|_{\mathcal{B}},
$$

and

$$
\left|f_{2}(n, x, y)-f_{2}(n, \bar{x}, \bar{y})\right| \leq a_{2}(n)\|x-\bar{x}\|_{\mathcal{B}}+b_{2}(n)\|y-\bar{y}\|_{\mathcal{B}}
$$

where $a_{i}, b_{i} \in l^{1}\left(\mathbb{Z}^{+}\right), i=1,2$;

$\left(C_{2}\right) f_{1}(\cdot, 0,0), f_{2}(\cdot, 0,0) \in l_{\delta}^{1}\left(\mathbb{Z}^{+}, \mathbb{C}^{r}\right) ;$

$\left(C_{3}\right)$ the matrix $\widehat{M} \in \mathcal{M}_{2 \times 2}\left(\mathbb{R}_{+}\right)$defined by

$$
\widehat{M}=\lambda d e^{-\delta}\left(\begin{array}{cc}
\left\|a_{1}\right\|_{1} & \left\|b_{1}\right\|_{1} \\
\left\|a_{2}\right\|_{1} & \left\|b_{2}\right\|_{1}
\end{array}\right)
$$

is convergent to zero.

Then, there is an unique weighted bounded solution $(x, y)$ of system (1.2) with $x_{0}=0, y_{0}=0$.

Proof. We define the operator $\Omega=\left(\Omega_{1}, \Omega_{2}\right)$ on $l_{\delta}^{\infty}\left(\mathbb{Z}^{+}, \mathcal{B}\right)$ by

$$
\Omega_{1}(\xi(n), \eta(n))=\sum_{s=0}^{n-1} U(n, s+1) f_{1}(s, \xi(s), \eta(s)), \xi, \eta \in l_{\delta}^{\infty}
$$

and

$$
\Omega_{2}(\xi(n), \eta(n))=\sum_{s=0}^{n-1} U(n, s+1) f_{2}(s, \xi(s), \eta(s)), \xi, \eta \in l_{\delta}^{\infty} .
$$

We now show that the operator $\Omega: l_{\delta}^{\infty}\left(\mathbb{Z}^{+}, \mathcal{B}\right) \times l_{\delta}^{\infty}\left(\mathbb{Z}^{+}, \mathcal{B}\right) \rightarrow l_{\delta}^{\infty}\left(\mathbb{Z}^{+}, \mathcal{B}\right) \times$ $l_{\delta}^{\infty}\left(\mathbb{Z}^{+}, \mathcal{B}\right)$ has a unique fixed point. We observe that $\Omega$ is well defined. In fact, we obtain

$$
\begin{aligned}
& \left\|\Omega_{1}(\xi, \eta)\right\|_{\mathcal{B}} e^{-\delta n} \\
& \leq \lambda d e^{-\delta} \sum_{s=0}^{n-1}\left|f_{1}(s, \xi(s), \eta(s))\right| e^{-\delta s} \\
& \leq \lambda d e^{-\delta}\left[\sum_{s=0}^{n-1}\left(a_{1}(s)\|\xi(s)\|_{\mathcal{B}} e^{\delta s}+b_{1}(s) e^{\delta s}\|\eta(s)\|_{\mathcal{B}}+\sum_{s=0}^{n-1}\left|f_{1}(\cdot, 0,0)\right| e^{\delta s}\right)\right]
\end{aligned}
$$


and

$$
\begin{aligned}
& \left\|\Omega_{2}(\xi, \eta)\right\|_{\mathcal{B}} e^{-\delta n} \\
& \leq \lambda d e^{-\delta} \sum_{s=0}^{n-1}\left|f_{2}(s, \xi(s), \eta(s))\right| e^{-\delta s} \\
& \leq \lambda d e^{-\delta}\left[\sum_{s=0}^{n-1}\left(a_{2}(s)\|\xi(s)\|_{\mathcal{B}} e^{\delta s}+b_{2}(s) e^{\delta s}\|\eta(s)\|_{\mathcal{B}}+\sum_{s=0}^{n-1}\left|f_{2}(\cdot, 0,0)\right| e^{\delta s}\right)\right],
\end{aligned}
$$

whence

$$
\left\|\Omega_{1}(\xi, \eta)\right\|_{\delta} \leq \lambda d e^{-\delta}\left[\left\|a_{1}\right\|_{1}\|\xi(s)\|_{\delta}+\left\|b_{1}\right\|_{1}\|\eta(s)\|_{\delta}+\left\|f_{1}(\cdot, 0,0)\right\|_{1, \delta}\right]
$$

and

$$
\left\|\Omega_{2}(\xi, \eta)\right\|_{\delta} \leq \lambda d e^{-\delta}\left[\left\|a_{2}\right\|_{1}\|\xi(s)\|_{\delta}+\left\|b_{2}\right\|_{1}\|\eta(s)\|_{\delta}+\left\|f_{2}(\cdot, 0,0)\right\|_{1, \delta}\right] .
$$

Hence, the space $l_{\beta}^{\infty}$ is invariant under $\Omega$. Next let $(\xi, \eta)$ and $(\bar{\xi}, \bar{\eta})$ be in $l_{\delta}^{\infty} \times l_{\delta}^{\infty}$. Then

$$
\left\|\Omega_{1}(\xi(s), \eta(s))-\Omega_{1}(\bar{\xi}(s), \bar{\eta}(s))\right\|_{\delta} \leq \lambda d e^{-\delta}\left[\left\|a_{1}\right\|_{1}\|\xi-\bar{\xi}\|_{\delta}+\left\|b_{1}\right\|_{1}\|\eta-\bar{\eta}\|_{\delta}\right]
$$

and

$\left\|\Omega_{2}(\xi(s), \eta(s))-\Omega_{2}(\bar{\xi}(s), \bar{\eta}(s))\right\|_{\delta} \leq \lambda d e^{-\delta}\left[\left\|a_{2}\right\|_{1}\|\xi-\bar{\xi}\|_{\delta}+\left\|b_{2}\right\|_{1}\|\eta-\bar{\eta}\|_{\delta}\right]$.

Then

$$
\|\Omega(\xi, \eta)-\Omega(\bar{\xi}, \bar{\eta})\|_{\delta} \leq \lambda d e^{-\delta}\left(\begin{array}{cc}
\left\|a_{1}\right\|_{1} & \left\|b_{1}\right\|_{1} \\
\left\|a_{2}\right\|_{1} & \left\|b_{2}\right\|_{1}
\end{array}\right)\left(\begin{array}{c}
\|\xi-\bar{\xi}\|_{\delta} \\
\|\eta-\bar{\eta}\|_{\delta}
\end{array}\right) .
$$

It follows that $\Omega$ has a unique fixed point $(\xi, \eta) \in l_{\delta}^{\infty}\left(\mathbb{Z}^{+}, \mathcal{B}\right) \times l_{\delta}^{\infty}\left(\mathbb{Z}^{+}, \mathcal{B}\right)$. The uniqueness of the solution is reduced to the uniqueness of the fixed point of the map $\Omega$. Let $(\xi, \eta)$ be the unique fixed point of $\Omega$. Then we have

$$
\|\xi\|_{\delta} \leq \lambda d e^{-\delta}\left[\left\|a_{1}\right\|_{1}\|\xi\|_{\delta}+\left\|b_{1}\right\|_{1}\|\eta\|_{\delta}+\left\|f_{1}(\cdot, 0,0)\right\|_{1, \delta}\right]
$$

and

$$
\|\eta\|_{\delta} \leq \lambda d e^{-\delta}\left[\left\|a_{2}\right\|_{1}\|\xi\|_{\delta}+\left\|b_{2}\right\|_{1}\|\eta\|_{\delta}+\left\|f_{2}(\cdot, 0,0)\right\|_{1, \delta}\right]
$$

Then

$$
\left(\begin{array}{c}
\|\xi\|_{\delta} \\
\|\eta\|_{\delta}
\end{array}\right) \leq \lambda d e^{-\delta}\left(\begin{array}{cc}
\left\|a_{1}\right\|_{1} & \left\|b_{1}\right\|_{1} \\
\left\|a_{2}\right\|_{1} & \left\|b_{2}\right\|_{1}
\end{array}\right)\left(\begin{array}{c}
\|\xi\|_{\delta} \\
\|\eta\|_{\delta}
\end{array}\right)+\lambda d e^{-\delta}\left(\begin{array}{c}
\left\|f_{1}(\cdot, 0,0)\right\|_{1, \delta} \\
\left\|f_{2}(\cdot, 0,0)\right\|_{1, \delta}
\end{array}\right) .
$$

So,

$$
\left(\begin{array}{c}
\|\xi\|_{\delta} \\
\|\eta\|_{\delta}
\end{array}\right) \leq \lambda d e^{-\delta}(I d-\widehat{M})^{-1}\left(\begin{array}{c}
\left\|f_{1}(\cdot, 0,0)\right\|_{1, \delta} \\
\left\|f_{2}(\cdot, 0,0)\right\|_{1, \delta}
\end{array}\right)
$$




\section{Asymptotic Periodicity}

The next result ensures the existence and uniqueness of a discrete Sasymptotically $\omega$-periodic solution for the problem (1.4).

THEOREM 6.1. Assume that the solution operator of (1.3) is strongly S-asymptotically $\omega$-periodic semigroup. Let $g_{1}, g_{2}: \mathbb{Z}^{+} \times \mathcal{B} \times \mathcal{B} \rightarrow \mathbb{C}^{r}$ be functions such that $g_{1}(\cdot, 0,0)$ and $g_{2}(\cdot, 0,0)$ are summable in $\mathbb{Z}^{+}$and there exist summable functions $a_{i}, b_{i} \in l^{1}\left(\mathbb{Z}^{+}\right), i=1,2$, such that

$$
\left|g_{1}(n, x, y)-g_{1}(n, \bar{x}, \bar{y})\right| \leq a_{1}(n)\|x-\bar{x}\|_{\mathcal{B}}+b_{1}(n)\|y-\bar{y}\|_{\mathcal{B}},
$$

and

$$
\left|g_{2}(n, x, y)-g_{2}(n, \bar{x}, \bar{y})\right| \leq a_{2}(n)\|x-\bar{x}\|_{\mathcal{B}}+b_{2}(n)\|y-\bar{y}\|_{\mathcal{B}},
$$

for all $x, y, \bar{x}, \bar{y} \in \mathcal{B}$ and $n \in \mathbb{Z}^{+}$. Then there is a unique discrete $S$ asymptotically $\omega$-periodic solution of the problem (1.4) for every $\varphi, \psi \in \mathcal{B}$.

Proof. We define the operator $T$ on the space $S A P_{\omega}(\mathcal{B})$ by

$$
T\left(\xi(n), \eta(n)=\left(T _ { 1 } \left(\xi(n), \eta(n), T_{2}(\xi(n), \eta(n)),\right.\right.\right.
$$

where

$$
T_{1}(\xi(n), \eta(n))=U(n) \varphi+\sum_{s=0}^{n-1} U(n-1-s) f_{1}(s, \xi(s), \eta(s)),
$$

for all $(\xi, \eta) \in S A P_{\omega}(\mathcal{B})$ and

$$
T_{2}(\xi(n), \eta(n))=U(n) \psi+\sum_{s=0}^{n-1} U(n-1-s) f_{2}(s, \xi(s), \eta(s)),
$$

for all $(\xi, \eta) \in S A P_{\omega}(\mathcal{B})$. Then we can write

$$
\nu_{1}(n)=\sum_{s=0}^{n-1} U(n-1-s) f_{1}(s, \xi(s), \eta(s)),
$$

for all $(\xi, \eta) \in S A P_{\omega}(\mathcal{B})$ and

$$
\nu_{2}(n)=\sum_{s=0}^{n-1} U(n-1-s) f_{2}(s, \xi(s), \eta(s)),
$$

for all $(\xi, \eta) \in S A P_{\omega}(\mathcal{B})$. We shall prove that $T$ is well defined. We note that the functions

$$
T(\cdot) \varphi, T(\cdot) \psi \in S A P_{\omega}(\mathcal{B}) .
$$

Moreover, the semigroup $U(n)$ is uniformly bounded in $\mathbb{Z}^{+}$. We get

$$
\left\|\nu_{1}\right\|_{\infty} \leq M d\left[\left\|a_{1}\right\|_{1}\|\xi\|_{\infty}+\left\|b_{1}\right\|_{1}\|\eta\|_{\infty}+\left\|f_{1}(., 0,0)\right\|_{1}\right]
$$

and

$$
\left\|\nu_{2}\right\|_{\infty} \leq M d\left[\left\|a_{2}\right\|_{1}\|\xi\|_{\infty}+\left\|b_{2}\right\|_{1}\|\eta\|_{\infty}+\left\|f_{2}(., 0,0)\right\|_{1}\right] .
$$


On the other hand, we have

$$
\begin{aligned}
& \left\|\sum_{s=n_{1}}^{m} U(m-s) f_{1}(s, \xi(s), \eta(s))\right\| \\
& \leq M\left[\left(\sum_{s=n_{1}}^{\infty} a_{1}(s)\right)\|\xi\|_{\infty}+\left(\sum_{s=n_{1}}^{\infty} b_{1}(s)\right)\|\eta\|_{\infty}+\left(\sum_{s=n_{1}}^{\infty}\left|f_{1}(s, 0,0)\right|\right)\right]
\end{aligned}
$$

and

$$
\begin{aligned}
& \left\|\sum_{s=n_{1}}^{m} U(m-s) f_{2}(s, \xi(s), \eta(s))\right\| \\
& \leq M\left[\left(\sum_{s=n_{1}}^{\infty} a_{2}(s)\right)\|\xi\|_{\infty}+\left(\sum_{s=n_{1}}^{\infty} b_{2}(s)\right)\|\eta\|_{\infty}+\left(\sum_{s=n_{1}}^{\infty}\left|f_{2}(s, 0,0)\right|\right)\right]
\end{aligned}
$$

Hence we obtain that

$$
\lim _{m \rightarrow \infty} \sum_{s=n_{1}}^{m} U(m-s) f_{1}(s, \xi(s), \eta(s))=0
$$

and

$$
\lim _{m \rightarrow \infty} \sum_{s=n_{1}}^{m} U(m-s) f_{2}(s, \xi(s), \eta(s))=0 .
$$

Taking into account that $T($.$) is S-asymptotically \omega$-periodic and

$$
\begin{aligned}
\nu_{1}(n+\omega)-\nu_{1}(n)= & \sum_{s=0}^{n_{1}-1}[U(n-1-s+\omega)-U(n-1-s)] f_{1}(s, \xi(s), \eta(s)) \\
& +\sum_{s=n_{1}}^{n_{1}-1+\omega} U(n-1-s+\omega) f_{1}(s, \xi(s), \eta(s)) \\
& -\sum_{s=n_{1}}^{n_{1}-1} U(n-1-s) f_{1}(s, \xi(s), \eta(s)),
\end{aligned}
$$

we obtain that

$$
\lim _{n \rightarrow \infty} \nu_{1}(n+\omega)-\nu_{1}(n)=0
$$

Similarly, we have

$$
\begin{aligned}
\nu_{2}(n+\omega)-\nu_{2}(n)= & \sum_{s=0}^{n_{1}-1}[U(n-1-s+\omega)-U(n-1-s)] f_{2}(s, \xi(s), \eta(s)) \\
& +\sum_{s=n_{1}}^{n_{1}-1+\omega} U(n-1-s+\omega) f_{2}(s, \xi(s), \eta(s))
\end{aligned}
$$




$$
-\sum_{s=n_{1}}^{n_{1}-1} U(n-1-s) f_{2}(s, \xi(s), \eta(s)),
$$

and hence

$$
\lim _{n \rightarrow \infty} \nu_{2}(n+\omega)-\nu_{2}(n)=0
$$

\section{Volterra Difference System with Infinite Delay}

We apply our previous result to Volterra difference systems with infinite delay. Volterra difference equations can be considered as natural generalization of difference equations. During the last few years Volterra difference equations have emerged vigorously in several applied fields, and currently there is wide interest in developing the qualitative theory for such equations.

Let $\gamma$ be a positive real number and let $A(n)$ and $K(n)$ be $r \times r$ matrices defined for $n \in \mathbb{Z}^{+}, s \in \mathbb{Z}^{+}$such that

$$
\sum_{n=0}^{\infty}|K(n)| e^{\gamma n}<+\infty
$$

and

$$
\|A\|_{\infty}=\sup _{n \geq 0}|A(n)|<\infty .
$$

We consider the following Volterra difference system with infinite delay

$$
x(n+1)=\sum_{s=-\infty}^{n} A(n) K(n-s) x(s), n \geq 0 .
$$

This equation is viewed as a functional difference equation on the phase space $\mathcal{B}_{\gamma}$, where $\mathcal{B}_{\gamma}$ is defined as follows:

$$
\mathcal{B}_{\gamma}=\mathcal{B}_{\gamma}\left(\mathbb{Z}^{-}, \mathbb{C}^{r}\right)=\left\{\varphi: \mathbb{Z}^{-} \rightarrow \mathbb{C}^{r}: \sup _{n \in \mathbb{Z}^{+}} \frac{\varphi(-n)}{e^{\gamma n}}<+\infty\right\}
$$

with the norm

$$
\|\varphi\|_{\mathcal{B}_{\gamma}}=\sup _{n \in \mathbb{Z}^{+}} \frac{\varphi(-n)}{e^{\gamma n}}, \varphi \in \mathcal{B}_{\gamma} .
$$

Next, we consider the following Volterra difference system with infinite delay

$$
\left\{\begin{aligned}
x(n+1) & =\sum_{s=-\infty}^{n} A(n) K(n-s) x(s)+a_{1}(n) x(n)+a_{2}(n) y(n), n \geq 0, \\
y(n+1) & =\sum_{s=-\infty}^{n} A(n) K(n-s) y(s)+b_{1}(n) x(n)+b_{2}(n) y(n), n \geq 0, \\
P(0) x_{0} & =\varphi \\
P(0) y_{0} & =\psi
\end{aligned}\right.
$$


We recall that the Volterra system (7.4) is viewed as retarded functional difference equations on the phase space $\mathcal{B}_{\gamma}$.

As consequence of Theorem 4.1 we have the following result.

THEOREM 7.1. Assume that system (7.1) has an exponential dichotomy, and $a_{i}, b_{i} \in l^{p}\left(\mathbb{Z}^{+}\right), i=1,2$. Then for each $\varphi, \psi \in P(0) \mathcal{B}_{\gamma}$ there is a unique bounded solution $(x, y)$ of the system (7.4) such that $(x, y.) \in l^{p}\left(\mathbb{Z}^{+}, \mathcal{B}_{\gamma}\right) \times$ $l^{p}\left(\mathbb{Z}^{+}, \mathcal{B}_{\gamma}\right)$; in particular, $(x, y) \in l^{p}\left(\mathbb{Z}^{+}, \mathbb{C}^{r}\right) \times l^{p}\left(\mathbb{Z}^{+}, \mathbb{C}^{r}\right)$.

Here

$$
\begin{aligned}
& f_{1}\left(n, x_{n}, y_{n}\right)=a_{1}(n) x(n)+a_{2}(n) y(n) ; \\
& f_{2}\left(n, x_{n}, y_{n}\right)=b_{1}(n) x(n)+b_{2}(n) y(n)
\end{aligned}
$$

and

$$
\begin{aligned}
& A_{1}\left(n, x_{n}, y_{n}\right)=\sum_{s=-\infty}^{n} A(n) K(n-s) x(s) ; \\
& A_{2}\left(n, x_{n}, y_{n}\right)=\sum_{s=-\infty}^{n} A(n) K(n-s) y(s) .
\end{aligned}
$$

Proof. Clearly, $\left\{A_{1}(n, \cdot, \cdot)\right\}$ and $\left\{A_{2}(n, \cdot, \cdot)\right\}$ are uniformly bounded sequences of bounded linear operators mapping $\mathcal{B} \times \mathcal{B}$ into $\mathbb{C}^{r}$. Here

$$
\left\|x_{n}\right\|_{\mathcal{B}_{\gamma}}=\sup _{s \in \mathbb{Z}^{+}} \frac{x_{n}(-s)}{e^{\gamma s}} \leq \sup _{s \in \mathbb{Z}^{+}}\|x(n-s)\| \leq \sup _{0 \leq s \leq n}\|x(s)\|,
$$

and the functions $f_{1}$ and $f_{2}$ satisfy: for all $x, y, \bar{x}, \bar{y} \in l^{p}\left(\mathbb{Z}^{+}, \mathcal{B}\right)$ and $n \in \mathbb{Z}^{+}$

$$
\begin{aligned}
\left|f_{1}(n, x, y)-f_{1}(n, \bar{x}, \bar{y})\right| & \leq a_{1}(n)\|x-\bar{x}\|_{p}+a_{2}(n)\|y-\bar{y}\|_{p}, \\
\left|f_{2}(n, x, y)-f_{2}(n, \bar{x}, \bar{y})\right| & \leq b_{1}(n)\|x-\bar{x}\|_{p}+b_{2}(n)\|y-\bar{y}\|_{p},
\end{aligned}
$$

where $a_{i}, b_{i} \in l^{p}\left(\mathbb{Z}^{+}\right), i=1,2$. Then

$$
M=2 d K\left(1-e^{-\alpha}\right)^{-1} \sup _{n \in \mathbb{Z}^{+}}\left(1+\|P(n)\|_{\mathcal{B}}\right)\left(\begin{array}{ll}
\left\|a_{1}\right\|_{p} & \left\|a_{2}\right\|_{p} \\
\left\|b_{1}\right\|_{p} & \left\|b_{2}\right\|_{p}
\end{array}\right) .
$$

Therefore, all the conditions of Theorem 4.1 are satisfied. If $M$ converges to zero, then problem (7.4) has a unique bounded solution $(x, y)$.

ACKNOWLEDGEMENTS.

This paper was completed while A. Ouahab visited the Department of Statistics, Mathematical Analysis and Optimization of the University of Santiago de Compostela. The work of J. J. Nieto has been partially supported by the AEI of Spain under Grant MTM2016- 75140-P and co-financed by European Community fund FEDER, and XUNTA de Galicia under grants GRC2015-004 and R2016/022.

The authors would like to thank the anonymous referees for their careful reading of the manuscript and pertinent comments; their constructive suggestions substantially improved the quality of the work. 


\section{REFERENCES}

[1] R. P. Agarwal, Difference equations and inequalities. Theory, methods and applications, Marcel Dekker, Inc., New York, 1992.

[2] R. P. Agarwal, C. Cuevas and M. Frasson, Semilinear functional difference equations with infinite delay, Math. Comput. Modelling 55 (2012), 1083-1105.

[3] R. P. Agarwal, D. O'Regan and P. J. Y. Wong, Constant-sign periodic and almost periodic solutions of a system of difference equations, Comput. Math. Appl. 50 (2005), 1725-1754.

[4] R. P. Agarwal and J. Popenda, Periodic solutions of first order linear difference equations, Math. Comput. Modelling 22 (1995), 11-19.

[5] R. P. Agarwal and P. J. Y. Wong, Advanced topics in difference equations, Kluwer Academic Publishers Group, Dordrecht, 1997.

[6] W. Arendt, Semigroups and evolution equations: functional calculus, regularity and kernel estimates, in Evolutionary equations. Vol. I, North-Holland, Amsterdam, 2004, $1-85$.

[7] C. T. H Baker and Y. Song, Periodic solutions of discrete Volterra equations, Math. Comput. Simulation 64 (2004), 521-542.

[8] S. Blunck, Analyticity and discrete maximal regularity on $L^{p}$-spaces, J. Funct. Anal. 183 (2001), 211-230.

[9] S. Blunck, Maximal regularity of discrete and continuous time evolution equations, Studia Math. 146 (2001), 157-176.

[10] A. Caicedo, C. Cuevas, G. M. Mophou, and G. M. N'Guérékata, Asymptotic behavior of solutions of some semilinear functional differential and integro-differential equations with infinite delay in Banach spaces, J. Franklin Inst. 349 (2012), 1-24.

[11] F. Cardoso and C. Cuevas, Exponential dichotomy and boundedness for retarded functional difference equations, J. Difference Equ. Appl. 15 (2009), 261-290.

[12] C. Corduneanu, Almost periodic discrete processes, Libertas Math. 2 (1982), 159-169.

[13] C. Cuevas, Weighted convergent and bounded solutions of Volterra difference systems with infinite delay, J. Differ. Equations Appl. 6 (2000), 461-480.

[14] C. Cuevas and L. Del Campo, An asymptotic theory for retarded functional difference equations, Comput. Math. Appl. 49 (2005), 841-855.

[15] C. Cuevas and M. Pinto, Convergent solutions of linear functional difference equations in phase space, J. Math. Anal. Appl. 277 (2003), 324-341.

[16] C. Cuevas, and C. Vidal, Discrete dichotomies and asymptotic behavior for abstract retarded functional difference equations in phase space, J. Difference Equ. Appl. 8 (2002), 603-640.

[17] C. Cuevas and C. Vidal, A note on discrete maximal regularity for functional difference equations with infinite delay, Adv. Difference Equ. (2006), Art. 97614, 1-11.

[18] B. De Andrade and C. Cuevas, S-asymptotically $\omega$-periodic and asymptotically $\omega$ periodic solutions to semi-linear Cauchy problems with non-dense domain, Nonlinear Anal. 72 (2010), 3190-3208.

[19] L. Del Campo, M. Pinto and C. Vidal, Almost and asymptotically almost periodic solutions of abstract retarded functional difference equations in phase space, J. Difference Equ. Appl. 17 (2011), 915-934.

[20] S. Elaydi, S. Murakami and E. Kamiyama, Asymptotic equivalence for difference equations with infinite delay, J. Differ. Equations Appl. 5 (1999), 1-23.

[21] A. Halanay, Solutions périodiques et presque-périodiques des systèmes d'équations aux differences finies, Arch. Rational Mech. Anal. 12 (1963), 134-149.

[22] Y. Hamaya, Existence of an almost periodic solution in a difference equation with infinite delay, J. Difference Equ. Appl. 9 (2003), 227-237. 
[23] H. R. Henríquez, M. Pierri and P. Táboas, On S-asymptotically $\omega$-periodic functions on Banach spaces and applications, J. Math. Anal. Appl. 343 (2008), 1119-1130.

[24] H. Matsunaga and S. Murakami, Some invariant manifolds for functional difference equations with infinite delay, J. Difference Equ. Appl. 10 (2004), 661-689.

[25] H. Matsunaga and S. Murakami, Asymptotic behavior of solutions of functional difference equations, J.Math. Anal. Appl. 305 (2005), 391-410.

[26] S. Murakami, Representation of solutions of linear functional difference equations in phase space, in Proceedings of the Second World Congress of Nonlinear Analysts, Part 2 (Athens, 1996), Nonlinear Anal. 30 (1997), 1153-1164.

[27] A. I. Perov, On the Cauchy problem for a system of ordinary differential equations, Pvibliž. Metod. Rešen Differencial. Uvavnen. Vyp. 2 (1964), 115-134 (in Russian).

[28] Y. Song, Periodic and almost periodic solutions of functional difference equations with finite delay, Adv. Difference Equ. (2007), Art. ID 68023, 15 pp.

[29] Y. Song, Asymptotically almost periodic solutions of nonlinear Volterra difference equations with unbounded delay, J. Difference Equ. Appl. 14 (2008), 971-986.

[30] Y. Song, Positive almost periodic solutions of nonlinear discrete systems with finite delay, Comput. Math. Appl. 58 (2009), 128-134.

[31] S. Sugiyama, On periodic solutions of difference equations, Bull. Sci. Engrg. Res. Lab. Waseda Univ. 52 (1971), 89-94.

[32] R. S. Varga, Matrix iterative analysis, Springer-Verlag, Berlin, 2000.

[33] C. Vidal, Existence of periodic and almost periodic solutions of abstract retarded functional difference equations in phase spaces, Adv. Difference Equ. (2009), Art. ID 380568, 19 pp.

\section{J. J. Nieto}

Instituto de Matemáticas, Facultade de Matemáticas

Universidade de Santiago de Compostela

Santiago de Compostela, 15782

Spain

E-mail: juanjose.nieto.roig@usc.es

A. Ouahab

Laboratory of Mathematics

University of Sidi Bel-Abbès

P.O. Box 89, 22000 Sidi Bel-Abbès

Algeria

E-mail: agh_ouahab@yahoo.fr

M. A. Slimani

Laboratory of Mathematics

University of Sidi Bel-Abbès

P.O. Box 89, 22000 Sidi Bel-Abbès

Algeria

E-mail: sedikslimani@yahoo.fr

Received: 15.9.2017.

Revised: 26.1.2018. 